\title{
Cooperative precoding design for cognitive wireless energy transfer networks
}

\author{
Tingting Zhang ${ }^{1}$, Yuanlian Huo ${ }^{1 \text { a) }}$, and Juan Zhao ${ }^{2}$ \\ ${ }^{1}$ College of Physics and Electronic Engineering, Northwest Normal University, \\ Anning, Lanzhou, Gansu 730-070, China \\ ${ }^{2}$ School of Telecommunication and Information Engineering, Nanjing University of \\ Posts and Telecommunications, Nanjing, Jiangsu 210-018, China
}

a)hylqqq@nwnu.edu.cn

\begin{abstract}
A cooperative precoding scheme is proposed for cognitive wireless energy transfer networks, where a multiple-input multiple-output multiple-antenna eavesdropper (MIMOME) primary link coexists with a multiantenna wireless energy transfer secondary link. Unlike the traditional wireless networks, the co-channel interference here is a useful rather than harmful resource. Our design objective is to maximize the secrecy rate of the primary link by optimizing the transmit covariance matrices, subject to the energy harvesting requirement at the energy receiver. The block coordinate descent (BCD)-based iterative algorithm is proposed to deal with the formulated non-convex optimization problem. Simulation results show that the proposed cooperative precoding design outperforms the non-cooperative scheme in terms of both secrecy rate and energy harvesting.
\end{abstract}

Keywords: wireless energy transfer, secrecy rate, coexisting, cooperative precoding

Classification: Wireless Communication Technologies

\section{References}

[1] Y. Zeng, B. Clerckx, and R. Zhang, "Communications and signals design for wireless power transmission," IEEE Trans. Commun., vol. 65, no. 5, pp. 2264 2290, May 2017. DOI:10.1109/TCOMM.2017.2676103

[2] C. Zhai, J. Liu, and L. Zheng, "Cooperative spectrum sharing with wireless energy harvesting in cognitive radio networks," IEEE Trans. Veh. Technol., vol. 65, no. 7, pp. 5303-5316, July 2016. DOI:10.1109/TVT.2015.2461447

[3] L. Jiang, H. Tian, C. Qin, S. Gjessing, and Y. Zhang, "Secure beamforming in wireless-powered cooperative cognitive radio networks," IEEE Commun. Lett., vol. 20, no. 3, pp. 522-525, Mar. 2016. DOI:10.1109/LCOMM.2016.2514353

[4] J. Xu, L. Liu, and R. Zhang, "Multiuser MISO beamforming for simultaneous wireless information and power transfer," IEEE Trans. Signal Process., vol. 62, no. 18, pp. 4798-4810, Sep. 2014. DOI:10.1109/TSP.2014.2340817

[5] Q. Shi, W. Xu, J. Wu, E. Song, and Y. Wang, "Secure beamforming for MIMO broadcasting with wireless information and power transfer," IEEE Trans. Wireless Commun., vol. 14, no. 5, pp. 2841-2853, May 2015. DOI:10.1109/ TWC.2015.2395414 


\section{Introduction}

Radio frequency (RF) signal enabled wireless energy transfer (WET) technique has attracted increasing attention recently due to its potential to solve the energy scarcity problem of energy-constrained wireless networks [1]. In practice, one WET system might be deployed in the same geographic region with some existing wireless communication systems such as cellular and WiFi networks. Therefore, a new design paradigm, namely, cognitive wireless energy transfer (CWET), has been proposed to improve the spectrum utilization efficiency of the overall network recently $[2,3]$. The key idea of CWET is to allow the WET secondary link to access the spectrum resource originally licensed to the wireless communication primary link opportunistically.

In this paper, we consider a secure CWET scenario, where a multiple-input multiple-output multiple-antenna eavesdropper (MIMOME) primary link coexists with a multi-antenna WET secondary link. The considered scenario is new and has not been studied in the existing literature yet. Since the MIMOME primary link and the WET secondary link share the same spectrum resource, the co-channel interference between these two links is unavoidable. For traditional wireless networks, interference is harmful because it will decrease the receiver's performance. However, it is worth noting that the co-channel interference here is useful rather than harmful. In particular, the co-channel interference generated by the energy transmitter (ET) of the secondary link can be considered as an artificial noise, which could be used to enhance the secrecy rate of the primary link. Meanwhile, the co-channel interference generated by the primary transmitter (PT) of the primary link is also an energy source for the energy receiver (ER) of the secondary link.

To make full use of the co-channel interference in the secure CWET network, in this paper, we study the cooperative precoding design problem. Specifically, we aim at maximizing the secrecy rate of the primary link while guaranteeing the energy harvesting constraint of the secrecy link by jointly optimizing the transmit covariance matrices of PT and ET. To solve the above-mentioned non-convex optimization problem, we propose a block coordinate descent (BCD)-based iterative algorithm with provable convergence. Numerical results show that the proposed precoding design outperforms the existing non-cooperative scheme in terms of both secrecy rate and energy harvesting.

\section{Proposed scheme}

Consider a secure CWET system, where a PT sends confidential messages to a primary user (PU) in the presence of an illegal eavesdropper (EA), and an ET of the secondary link transmits energy signal to a ER in a wireless manner with the same spectrum resource authorized to the PU. The number of antennas employed by PT, PU, EA, ET, and ER are $N_{t}, N_{p}, N_{e}, N_{s}$, and $N_{h}$, respectively.

The co-channel interference generated by the ET can be regarded as artificial noise to help PU to achieve secure communication. Therefore, the secrecy rate of the primary link is given by

$$
R(\mathbf{Q}, \mathbf{S})=R_{p}(\mathbf{Q}, \mathbf{S})-R_{e}(\mathbf{Q}, \mathbf{S})
$$


where $R_{p}(\mathbf{Q}, \mathbf{S})=\log \left|\sigma_{p}^{2} \mathbf{I}+\mathbf{G}_{p} \mathbf{S G}_{p}^{H}+\mathbf{H}_{p} \mathbf{Q} \mathbf{H}_{p}^{H}\right|-\log \left|\sigma_{p}^{2} \mathbf{I}+\mathbf{G}_{p} \mathbf{S G}_{p}^{H}\right| \quad$ is the achievable rate of the $\mathrm{PU} ; \quad R_{e}(\mathbf{Q}, \mathbf{S})=\log \left|\sigma_{e}^{2} \mathbf{I}+\mathbf{G}_{e} \mathbf{S G}_{e}^{H}+\mathbf{H}_{e} \mathbf{Q} \mathbf{H}_{e}^{H}\right|-$ $\log \left|\sigma_{e}^{2} \mathbf{I}+\mathbf{G}_{e} \mathbf{S} \mathbf{G}_{e}^{H}\right|$ is the achievable rate of the EA; $\mathbf{Q}$ and $\mathbf{S}$ are the transmit covariance matrices of the PT and the ET, respectively, which are to be designed; $\mathbf{H}_{p} \in \mathbb{C}^{N_{p} \times N_{t}}$ and $\mathbf{H}_{e} \in \mathbb{C}^{N_{e} \times N_{t}}$ respectively denote the channel matrices from the PT to the PU and the eavesdropper; $\mathbf{G}_{p} \in \mathbb{C}^{N_{p} \times N_{s}}$ and $\mathbf{G}_{e} \in \mathbb{C}^{N_{e} \times N_{s}}$ are the channel matrices from the ET to the PU and the eavesdropper, respectively; $\sigma_{p}^{2}$ and $\sigma_{e}^{2}$ denote the noise power at the PU and the eavesdropper, respectively;

On the other hand, the co-channel interference generated by the PT can be regarded as an energy source for the ER. Hence, the total harvested energy at the ER is expressed as

$$
E(\mathbf{Q}, \mathbf{S})=\eta\left(\operatorname{Tr}\left(\mathbf{G}_{h} \mathbf{S G}_{h}^{H}\right)+\operatorname{Tr}\left(\mathbf{H}_{h} \mathbf{Q} \mathbf{H}_{h}^{H}\right)\right)
$$

where $0 \leq \eta \leq 1$ denotes the energy harvesting efficiency, $\mathbf{G}_{h} \in \mathbb{C}^{N_{h} \times N_{s}}$ and $\mathbf{H}_{h} \in$ $\mathbb{C}^{N_{h} \times N_{t}}$ represent the channel matrices from the ET and the PT to the ER, respectively.

The ET would send energy signal for enhancing the secure transmission of the primary link under the condition that its energy harvesting target can be satisfied. Therefore, our design objective is to maximize the secrecy rate of the primary link by optimizing the transmit covariance matrices of PT and ET, subject to the energy harvesting constraint at the ER. Mathematically, the above-mentioned problem can be formulated as

$$
\begin{aligned}
& \max _{\mathbf{Q} \geq 0, \mathbf{S} \geq 0} R(\mathbf{Q}, \mathbf{S}) \\
& \text { s.t. } E(\mathbf{Q}, \mathbf{S}) \geq e_{0}, \operatorname{Tr}(\mathbf{Q}) \leq p_{t}, \operatorname{Tr}(\mathbf{S}) \leq p_{s}
\end{aligned}
$$

where $e_{0}$ is energy harvesting target of the ER; $p_{t}$ and $p_{s}$ are the maximum transmit power at PT and ET, respectively. Note that problem (3) is non-convex due to the complicated objective function, and it is difficult to solve in general. To deal with the non-convex problem (3), in the following, we first reformulate problem (3) to an equivalent problem and then propose a BCD-based iterative algorithm to tackle the resulting problem.

\section{BCD-based iterative algorithm for problem (3)}

Prior to solving problem (3), we first have a check on its feasibility, i.e., whether a given energy harvesting target $e_{0}$ for the ER can be met under the given transmit power $p_{t}$ and $p_{s}$ constraints. To this end, we need to solve the following problem.

$$
\begin{gathered}
E^{*}=\max _{\mathbf{Q} \geq 0, \mathbf{S} \geq 0} \eta\left(\operatorname{Tr}\left(\mathbf{G}_{h} \mathbf{S} \mathbf{G}_{h}^{H}\right)+\operatorname{Tr}\left(\mathbf{H}_{h} \mathbf{Q} \mathbf{H}_{h}^{H}\right)\right) \\
\text { s.t. } \operatorname{Tr}(\mathbf{Q}) \leq p_{t}, \operatorname{Tr}(\mathbf{S}) \leq p_{s}
\end{gathered}
$$

It is noted that problem (4) is a convex optimization problem. According to [4], it is easily obtained that the optimal value of problem (4) is $E^{*}=$ $\eta\left(p_{t} \lambda_{\max }\left(\mathbf{H}_{h}^{H} \mathbf{H}_{h}\right)+p_{s} \lambda_{\max }\left(\mathbf{G}_{h}^{H} \mathbf{G}_{h}\right)\right)$, where $\lambda_{\max }(\cdot)$ denotes the maximum eigenvalue of a matrix. Therefore, the feasibility of the original problem (3) for a given $e_{0}$, $p_{t}$, and $p_{s}$ can be easily verified by checking whether $e_{0} \leq E^{*}$. Without loss of generality, in the rest of this paper, we assume that problem (3) is always feasible. 
In the following, we will transform the problem (3) to an equivalent problem, which is more easier to tackle by using the BCD approach. For this purpose, we need the following lemma [5].

Lemma 1 Let $\mathbf{U} \in \mathbb{C}^{N \times N}$ be any matrix such that $\mathbf{U} \geq \mathbf{0}$. Consider the function $f(\mathbf{W})=-\operatorname{Tr}(\mathbf{W} \mathbf{U})+\log |\mathbf{W}|+N$. Then,

$$
-\log |\mathbf{U}|=\max _{\mathbf{W} \in \mathbb{C}^{N \times N}, \mathbf{W} \geq 0} f(\mathbf{W}),
$$

and the optimal solution to the right-hand side of (5) is $\mathbf{W}^{*}=\mathbf{U}^{-1}$.

Therefore, according to Lemma 1 , we can equivalently re-express the term $-\log \left|\sigma_{p}^{2} \mathbf{I}+\mathbf{G}_{p} \mathbf{S} \mathbf{G}_{p}^{H}\right|$ in $R_{p}(\mathbf{Q}, \mathbf{S})$ as

$$
-\log \left|\sigma_{p}^{2} \mathbf{I}+\mathbf{G}_{p} \mathbf{S} \mathbf{G}_{p}^{H}\right|=\max _{\mathbf{W}_{1} \geq 0} \log \left|\mathbf{W}_{1}\right|-\operatorname{Tr}\left(\mathbf{W}_{1}\left(\sigma_{\mathrm{p}}^{2} \mathbf{I}+\mathbf{G}_{\mathrm{p}} \mathbf{S G}_{\mathrm{p}}^{\mathrm{H}}\right)\right)+N_{p}
$$

Similarly, we have

$$
\begin{aligned}
- & \log \left|\sigma_{e}^{2} \mathbf{I}+\mathbf{G}_{e} \mathbf{S} \mathbf{G}_{e}^{H}+\mathbf{H}_{e} \mathbf{Q} \mathbf{H}_{e}^{H}\right| \\
& =\max _{\mathbf{W}_{2} \geq 0} \log \left|\mathbf{W}_{2}\right|-\operatorname{Tr}\left(\mathbf{W}_{2}\left(\sigma_{\mathrm{e}}^{2} \mathbf{I}+\mathbf{G}_{\mathrm{e}} \mathbf{S} \mathbf{G}_{\mathrm{e}}^{\mathrm{H}}+\mathbf{H}_{\mathrm{e}} \mathbf{Q} \mathbf{H}_{\mathrm{e}}^{\mathrm{H}}\right)\right)+N_{e}
\end{aligned}
$$

Substituting (6) and (7) into (1), we can then reformulate problem (3) as

$$
\begin{aligned}
& \max _{\mathbf{Q} \geq 0, \mathbf{S} \geq 0, \mathbf{W}_{1} \geq 0, \mathbf{W}_{2} \geq 0} \bar{R}\left(\mathbf{Q}, \mathbf{S}, \mathbf{W}_{1}, \mathbf{W}_{2}\right) \\
& \text { s.t. } E(\mathbf{Q}, \mathbf{S}) \geq e_{0}, \operatorname{Tr}(\mathbf{Q}) \leq p_{t}, \operatorname{Tr}(\mathbf{S}) \leq p_{s}
\end{aligned}
$$

where $\bar{R}\left(\mathbf{Q}, \mathbf{S}, \mathbf{W}_{1}, \mathbf{W}_{2}\right)=\log \left|\sigma_{p}^{2} \mathbf{I}+\mathbf{G}_{p} \mathbf{S} \mathbf{G}_{p}^{H}+\mathbf{H}_{p} \mathbf{Q} \mathbf{H}_{p}^{H}\right|+\log \left|\mathbf{W}_{1}\right|+\log \mid \sigma_{e}^{2} \mathbf{I}+$ $\mathbf{G}_{e} \mathbf{S G}_{e}^{H}|+\log | \mathbf{W}_{2} \mid-\operatorname{Tr}\left(\mathbf{W}_{2}\left(\sigma_{e}^{2} \mathbf{I}+\mathbf{G}_{e} \mathbf{S G}_{e}^{H}+\mathbf{H}_{e} \mathbf{Q} \mathbf{H}_{e}^{H}\right)\right)-\operatorname{Tr}\left(\mathbf{W}_{1}\left(\sigma_{p}^{2} \mathbf{I}+\right.\right.$ $\left.\left.\mathbf{G}_{p} \mathbf{S G}_{p}^{H}\right)\right)+N_{e}+N_{p}$.

It is noted that problem (8) is not jointly concave with respect to variables $\left\{\mathbf{Q}, \mathbf{S}, \mathbf{W}_{1}, \mathbf{W}_{2}\right\}$. However, it is concave with respect to $\{\mathbf{Q}, \mathbf{S}\}$ for a fixed $\left\{\mathbf{W}_{1}, \mathbf{W}_{2}\right\}$ and vice versa. This coordinate-wise convexity property motives us to use BCD method to tackle problem (8). Specifically, we need to solve the following three subproblems in an alternating fashion.

$$
\begin{gathered}
\mathbf{W}_{1}^{n}=\arg \max _{\mathbf{W}_{1} \geq 0} \log \left|\mathbf{W}_{1}\right|-\operatorname{Tr}\left(\mathbf{W}_{1}\left(\sigma_{p}^{2} \mathbf{I}+\mathbf{G}_{p} \mathbf{S}^{n-1} \mathbf{G}_{p}^{H}\right)\right) \\
\mathbf{W}_{2}^{n}=\arg \max _{\mathbf{W}_{2} \geq 0} \log \left|\mathbf{W}_{2}\right|-\operatorname{Tr}\left(\mathbf{W}_{2}\left(\sigma_{e}^{2} \mathbf{I}+\mathbf{G}_{e} \mathbf{S}^{n-1} \mathbf{G}_{e}^{H}+\mathbf{H}_{e} \mathbf{Q}^{n-1} \mathbf{H}_{e}^{H}\right)\right) \\
\left(\mathbf{Q}^{n}, \mathbf{S}^{n}\right)=\arg \max _{\mathbf{Q} \geq 0, \mathbf{S} \geq 0} \bar{R}\left(\mathbf{Q}, \mathbf{S}, \mathbf{W}_{1}^{n}, \mathbf{W}_{2}^{n}\right) \\
\text { s.t. } E(\mathbf{Q}, \mathbf{S}) \geq e_{0}, \operatorname{Tr}(\mathbf{Q}) \leq p_{t}, \operatorname{Tr}(\mathbf{S}) \leq p_{s}
\end{gathered}
$$

where $n$ denotes the $n$th iteration.

According to Lemma 1, the optimal solutions of problem (9) and problem (10) with a fixed $\left\{\mathbf{Q}^{n-1}, \mathbf{S}^{n-1}\right\}$ are respectively given by

$$
\begin{gathered}
\mathbf{W}_{1}^{n}=\left(\sigma_{p}^{2} \mathbf{I}+\mathbf{G}_{p} \mathbf{S}^{n-1} \mathbf{G}_{p}^{H}\right)^{-1} \\
\mathbf{W}_{2}^{n}=\left(\sigma_{e}^{2} \mathbf{I}+\mathbf{G}_{e} \mathbf{S}^{n-1} \mathbf{G}_{e}^{H}+\mathbf{H}_{e} \mathbf{Q}^{n-1} \mathbf{H}_{e}^{H}\right)^{-1}
\end{gathered}
$$

Note that problem (11) is a convex optimization problem and thus can be efficiently solved by interior-point method using existing software, e.g., CVX.

Based on the discussion above, we now readily summarize our proposed approach to solve problem (3) as Algorithm 1 shown in Table I. 
Table I. BCD-based iterative algorithm for problem (3)

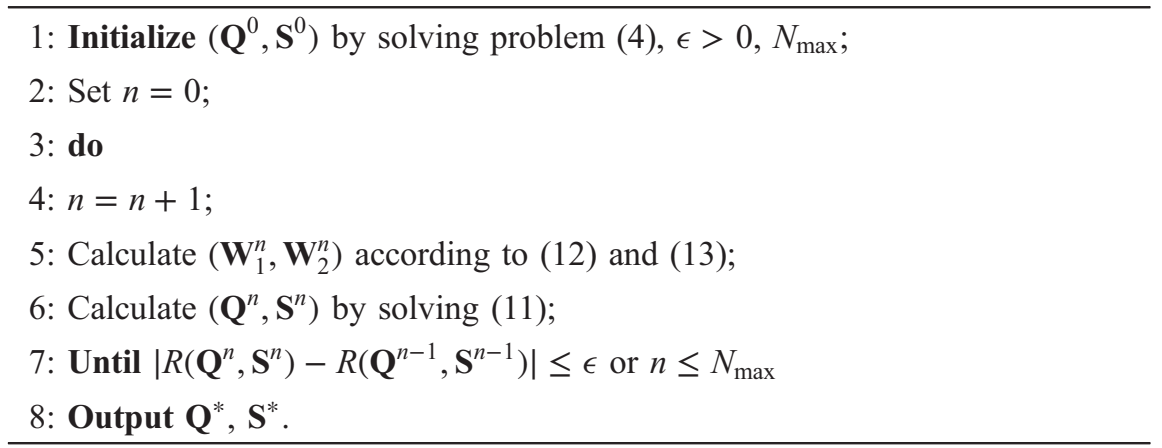

One can easily verify that the proposed algorithm leads to a non-descending objective values, i.e., $R\left(\mathbf{Q}^{n}, \mathbf{S}^{n}\right) \geq R\left(\mathbf{Q}^{n-1}, \mathbf{S}^{n-1}\right) \geq \ldots \geq R\left(\mathbf{Q}^{0}, \mathbf{S}^{0}\right)$. Meanwhile, the total transmit power is limited. Therefore, the proposed algorithm will monotonically converge.

\section{Simulation results}

In this section, simulation results are presented to validate the performance of our proposed algorithm. In our examples, we set simulation parameters as follows: $N_{t}=N_{s}=5, \quad N_{p}=N_{e}=N_{h}=2, \quad \eta=0.6, \quad p_{t}=p_{s}=30 \mathrm{dBm}, \quad \sigma_{p}^{2}=\sigma_{e}^{2}=-70$ $\mathrm{dBm}$. The channels are assumed to be quasi-static flat Rayleigh fading and independent of each other. Also, each channel has i.i.d. entries distributed as $\mathcal{C N}(0,-40 \mathrm{~dB})$, where $-40 \mathrm{~dB}$ corresponds to the signal attenuation from each transmitter to all receivers.

Fig. 1 shows the convergence behavior of our proposed algorithm for different channel realizations with $e_{0}=-5 \mathrm{dBm}$. It can be observed that the total transmit power monotonically decreases and finally achieves convergence within 10 iterations, which reveals that the proposed algorithm has a fast convergence rate.

Fig. 2 compares the performance of our proposed cooperative precoding design with that of the non-cooperative baseline scheme. For the baseline scheme, there is no cooperation between the PT and the ET. From Fig. 2, we can see that our proposed precoding design outperforms the non-cooperative baseline scheme in terms of both secrecy rate and energy harvesting. Moreover, we can also notice that the secrecy rate gradually decreases as the energy harvesting requirement increases.

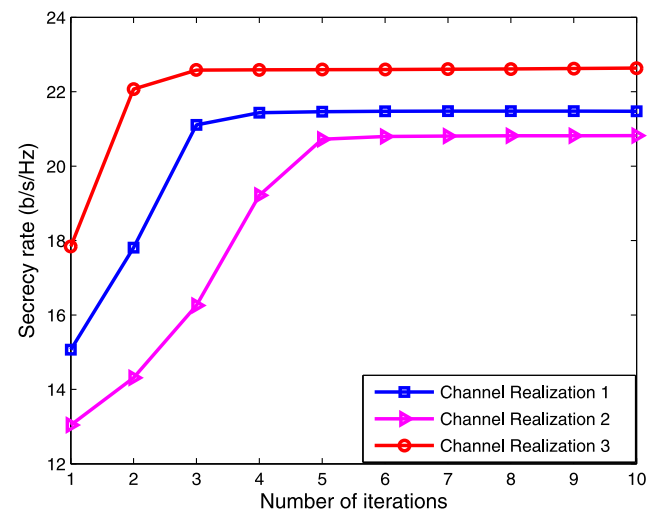




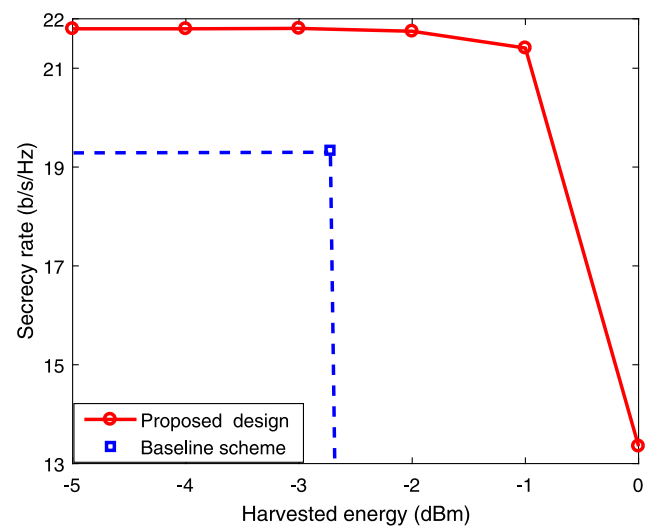

Fig. 2. Secrecy rate versus harvested energy.

\section{Conclusion}

In this paper, we have studied the cooperative precoding design for a secure CWET system. The design objective is to maximize the secrecy rate subject to the energy harvesting requirement. We have proposed a BCD-based iterative to deal with the original non-convex optimization problem. Simulation results show that the proposed precoding design outperforms the non-cooperative design in terms of both secrecy rate and energy harvesting.

\section{Acknowledgments}

This work was supported by the National Natural Science Foundation of China under Grants 61561044. 